\title{
Criteria for clinical stability in hospitalised patients with community-acquired pneumonia
}

\author{
Stefano Aliberti1,2, Anna Maria Zanaboni³, Tim Wiemken², Ahmed Nahas², \\ Srinivas Uppatlaㄹ, Letizia Corinna Morlacchi ${ }^{4}$, Paula Peyrani ${ }^{2}$, Francesco Blasi ${ }^{4}$ \\ and Julio Ramirez ${ }^{2}$
}

Affiliations: 'Dept of Health Science, University of Milan Bicocca, AO San Gerardo, Monza, ${ }^{3}$ Computer Science Dept, University of Milan, Milan, and ${ }^{4}$ Dipartimento di Fisiopatologia Medico-Chirurgica e dei Trapianti, University of Milan, IRCCS Fondazione Cà Granda Policlinico, Milan, Italy. ${ }^{2}$ Division of Infectious Diseases, Dept of Medicine, University of Louisville, Louisville, KY, USA.

Correspondence: S. Aliberti, Dept. of Health Science, University of Milan Bicocca, Respiratory Unit, AO San Gerardo, Via Pergolesi 33, 20052, Monza, Italy. E-mail: stefano.alibertidunimib.it

ABSTRACT The American Thoracic Society (ATS) and Infectious Diseases Society of America (IDSA) suggested two sets of criteria in 2001 and 2007 to define clinical stability in community-acquired pneumonia (CAP). The present study aimed to evaluate the level of agreement between these two sets of criteria and how well they can predict clinical outcomes.

A retrospective cohort study was carried out of 487 consecutive patients hospitalised with CAP. Level of agreement was tested using a survival curve analysis, while prediction of outcomes at 30-day follow-up was evaluated through receiver operating characteristic (ROC) analysis.

A discrepancy between ATS 2001 and ATS/IDSA 2007 criteria in identifying clinical stability was detected in $62 \%$ of the patients. The median (interquartile range) time to clinical stability was 2 (1-4) days based on ATS 2001 and $3(2-5)$ days based on ATS/IDSA 2007 criteria $(p=0.012)$. The daily distribution of patients who reached clinical stability evaluated with both sets was different $(p=0.002)$. The ROC analysis showed an area under the curve of 0.705 for the ATS 2001 criteria and 0.714 for ATS/IDSA 2007 criteria $(p=0.645)$.

ATS 2001 and ATS/IDSA 2007 criteria for clinical stability in hospitalised patients with CAP are clinically equivalent and both can be used in clinical practice as well as in clinical research.

@ERSpublications

ATS 2001 and ATS/IDSA 2007 criteria for clinical stability in hospitalised patients with CAP are clinically equivalent http://ow.ly/lAeKE

Received: June 292012 | Accepted after revision: Oct 222012 | First published online: Nov 082012

Conflict of interest: None declared.

Copyright @ERS 2013 


\section{Introduction}

Community-acquired pneumonia (CAP) occurs in up to 5.6 million patients every year in the USA and more than 1 million require hospitalisation [1]. After initiation of appropriate empirical antibiotic therapy, the majority of patients who are hospitalised show evidence of clinical improvement. The process of clinical improvement starts when a patient with CAP becomes clinically stable. Identification of clinical stability has several implications in clinical practice and clinical research. In clinical practice, time to clinical stability is used to guide the switch from i.v. to oral antibiotic therapy and it can also help to define the time for hospital discharge, as well as adverse events after hospitalisation [2, 3]. In clinical trials, patient's clinical stability is an early outcome used to compare antibiotic treatments in CAP patients. Recently, the US Food and Drug Administration (FDA) acknowledged clinical stability as one of the most important end-points for patients with bacterial pneumonia [4].

Although a general agreement exists on the importance of the recognition of clinical stability as an early outcome in patients with CAP, there is surprisingly little scientific literature on how its evaluation should be performed [5]. Guidelines on the management of CAP published by the American Thoracic Society (ATS) in 2001 suggested a definition of clinical stability that was based on patient symptoms, such as cough and shortness of breath, along with signs of systemic response, such as fever and white blood cell count [1]. These criteria were initially reported by RAMIREZ et al. [2] in 1995. The latest ATS and the Infectious Diseases Society of America (IDSA) guidelines, published in 2007, define clinical stability as a decrease under fixed thresholds of vital parameters, including temperature, heart rate, respiratory rate, blood pressure, mental status and oxygenation [6]. This new approach developed from physician-based observations, as originally described by HaLm et al. [7] in 1998.

The ATS 2001 criteria are based on patient symptomatology, while the ATS/IDSA 2007 definition of clinical stability is based on objective parameters. Although these are the two most common criteria used to define clinical stability, they have never been evaluated in a head-to-head comparison in the same population of hospitalised patients with CAP.

The aim of our study was to compare the ATS 2001 and ATS/IDSA 2007 criteria for clinical stability in hospitalised patients with CAP in an attempt to: 1) define the level of agreement between the two sets of criteria in relation to time to clinical stability; and 2) define how well these criteria can identify patients with good clinical outcome during a 30-day follow-up period.

\section{Material and methods \\ Study design}

This was an observational, retrospective study of consecutive patients admitted with a diagnosis of CAP at the Veterans Administration Medical Center (VAMC) in Louisville, KY, USA, between June 2001 and March 2006. Patients enrolled in this study are part of the Community-Acquired Pneumonia Organization database [8]. The study protocol, data collection form and all the completed data collection forms are available on the study website (www.caposite.com). Details about data collection and management have been described previously [9]. The institutional review board of the VAMC approved the study (human study subcommittee; CAPO IRB \#0061).

\section{Study population and measurements}

Patients $\geqslant 18$ years of age who met the criteria for CAP were included in the study. Patients in whom findings to identify clinical stability were missing were excluded from the study. The records of all enrolled patients were reviewed. Data from the VAMC system were extracted pertaining to: patient demographics; medical comorbidities; clinical and laboratory variables; radiographic findings; the pneumonia severity index; the CURB-65 (confusion, urea $>7 \mathrm{mmol} \cdot \mathrm{L}^{-1}$, respiratory rate $\geqslant 30$ breaths $\cdot \mathrm{min}^{-1}$, blood pressure $<90 \mathrm{mmHg}$ (systolic) $\leqslant 60 \mathrm{mmHg}$ (diastolic), age $\geqslant 65$ years) score; microbiological and in-hospital treatment data; clinical stability; length of hospital stay; adverse outcomes during hospitalisation and within 30 days after hospital discharge [10, 11]. Two investigators (A Nahas and S Uppatla.) independently reviewed the electronic charts of all the enrolled patients and collected data on clinical stability.

\section{Study definitions}

CAP was defined as the presence of a new pulmonary infiltrate on chest radiograph at the time of hospitalisation associated with at least one of the following: 1) new or increased cough; 2) an abnormal temperature $\left(<35.6^{\circ} \mathrm{C}\right.$ or $\left.\left.>37.8^{\circ} \mathrm{C}\right) ; 3\right)$ an abnormal serum leukocyte count (leukocytosis, left shift, or leukopenia defined by local laboratory values). Severe CAP was defined based on the ATS/IDSA guidelines published in 2007 [6]. 
TABLE 1 American Thoracic Society (ATS) 2001 and ATS/Infectious Diseases Society of America (IDSA) 2007 criteria for clinical stability in hospitalised patients with communityacquired pneumonia

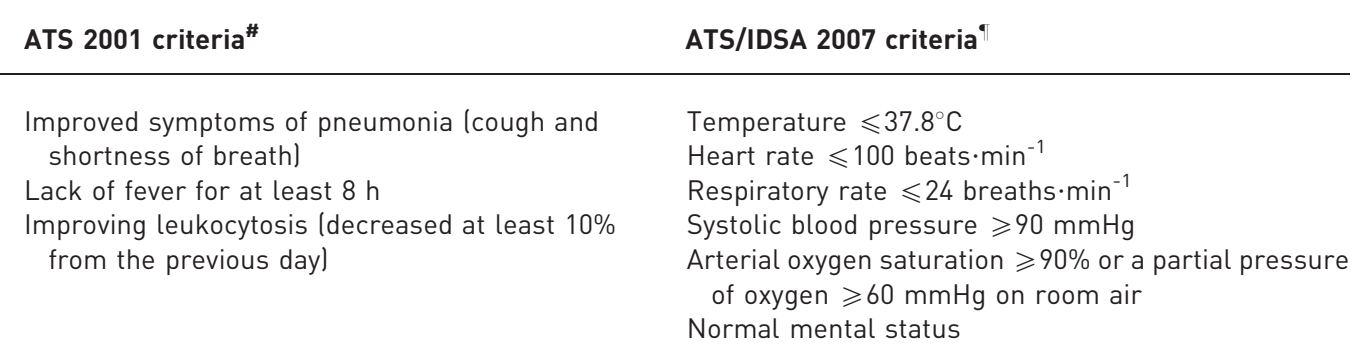

\#: all the criteria should be present during the same day in comparison to the previous day to define clinical stability; ${ }^{\circ}$ : all the criteria should be present during the same day to define clinical stability.

Criteria to define clinical stability were evaluated daily during the first 7 days of hospitalisation. Clinical stability was defined according to two criteria: ATS guidelines published in 2001 and ATS/IDSA guidelines published in 2007 [1, 6]. Table 1 depicts the two sets of criteria for clinical stability. Time to clinical stability was calculated as the number of days from the date of hospital admission to the date that the patient met clinical stability criteria. Length of hospital stay was calculated as the number of days from the date of admission to the date of discharge. Length of hospital stay was censored at 14 days in an effort to capture only CAP-related length of hospital stay.

\section{Outcome definition}

The presence of clinical failure either during hospitalisation or after hospital discharge was defined as the outcome of interest. Clinical failure during hospitalisation was considered if any of the following took place after patient admission to the floor and initial stabilisation: 1) acute pulmonary deterioration with the need for either invasive or noninvasive mechanical ventilation; 2) acute haemodynamic deterioration with the need for aggressive fluid resuscitation (approximately $40 \mathrm{~mL} \cdot \mathrm{kg}^{-1}$ of colloids or crystalloids), vasopressors or invasive procedures (e.g. pericardial drainage, electrical cardioversion); and 3) in-hospital death up to 28 days after admission. Clinical failure after discharge was defined as either readmission or death for any reason within 30 days after hospital discharge.

\section{Comparison between ATS 2001 and ATS/IDSA 2007}

The level of agreement between ATS 2001 and ATS/IDSA 2007 criteria was detected by the evaluation of the joint distribution of time to clinical stability in the study population according to both scores. Survival curve analysis was used to compare between the two scores the daily distribution of patients who reached clinical stability during the first week of hospitalisation.

ATS 2001 and ATS/IDSA 2007 criteria for clinical stability with respect to clinical outcome were compared through receiver operating characteristic (ROC) analysis of their predictive value with respect to clinical failure, either during hospitalisation or after hospital discharge.

\section{Statistical analysis}

All data were statistically analysed using SPSS (version 18.0) for Mac (IBM, New York, NY, USA). Descriptive statistics were reported at baseline, with continuous data expressed as a median (interquartile range) and categorical data expressed as frequencies and percentages. A generalised Wilcoxon test was performed to compare the survival curves (Kaplan-Meier). The difference of median (interquartile range) time to clinical stability according to the ATS 2001 and ATS/IDSA 2007 criteria was evaluated by the Wilcoxon-Mann-Whitney U two-sample test. The predictive value of the ATS 2001 and ATS/IDSA 2007 criteria for clinical stability was explored for indicating the presence of a clinical failure via a ROC curve. Each point of the ROC curve corresponds to a specific time to clinical stability value (e.g. $t$ days). The coordinates of each point of the ROC curve were computed as follows: the sensitivity of time to clinical stability was calculated as the frequency of patients who did not reach clinical stability within t days among those who had a clinical failure The specificity of time to clinical stability was calculated as the frequency of patients who reached clinical stability within $t$ days among those who did not have a clinical failure. The difference in areas under the curve (AUC) was tested according to DeLong et al. [12]. All tests were twotailed and a p-value $<0.05$ was considered statistically significant. 


\section{Results}

Study population

A total of 500 consecutive patients with CAP were enrolled during the study period. The final study population consisted of 487 patients, due to lack of data in 13 patients. Characteristics of the study population are summarised in table 2 . Among the entire study population, median (interquartile range) length of stay in the hospital was 4 (3-7) days. A total of 64 patients (13\%) experienced a clinical failure during hospitalisation: $39(8 \%)$ had a respiratory instability, $10(2 \%)$ had haemodynamic instability and 33 (7\%) died. A total of 71 patients (15\%) experienced a clinical failure 30 days after discharge: 71 (15\%) were re-hospitalised and $17(3.5 \%)$ died.

\section{Level of agreement between ATS 2001 and ATS/IDSA 2007 criteria}

After initiation of empiric antibiotic therapy, a total of 429 patients (88\%) reached clinical stability during the first 7 days of hospitalisation according to the ATS 2001 criteria and 410 patients (84\%) according to the ATS/IDSA 2007 criteria. The level of agreement between the two sets of criteria with regard to time to clinical stability detected in the study population is shown in table 3. A discrepancy between ATS 2001 and ATS/IDSA 2007 criteria in identifying clinical stability within the first week of hospitalisation was detected in 301 patients $(62 \%)$.

Among those who reached clinical stability within the first week of hospitalisation, the median (interquartile range) time to clinical stability was 2 (1-4) days based on ATS 2001 criteria and 3 (2-5) days based on ATS/IDSA 2007 criteria $(p=0.012)$. ATS 2001 criteria identified clinical stability earlier than ATS/ IDSA 2007 criteria in $40 \%$ of the study population. Clinical stability was reached 1 day earlier based on ATS 2001 criteria (in comparison to ATS/IDSA 2007 criteria) in 23\% of the population, 2 days earlier in $11 \%$ of the population, and 3 days earlier in $4 \%$ of the population. The daily distribution of patients who reached clinical stability during the first week of hospitalisation is depicted in figure 1, showing a significant difference with regard to ATS 2001 and ATS/IDSA 2007 criteria (Breslow generalised Wilcoxon; p=0.002).

\section{ATS 2001 versus ATS/IDSA 2007 criteria with respect to clinical outcomes}

A total of 11 patients $(2.6 \%)$ experienced a clinical failure during hospitalisation after reaching clinical stability according to the ATS 2001 criteria and 69 patients (16\%) experienced a clinical failure after discharge. A total of five patients (1.2\%) experienced a clinical failure during hospitalisation after reaching clinical stability according to the ATS 2007 criteria and 64 patients (16\%) experienced a clinical failure after discharge.

Table 4 depicts clinical outcomes both during hospitalisation and 30 days after discharge among those who reached clinical stability within the first week of hospitalisation according to both scores. Among the 429 patients who reached clinical stability within the first week of hospitalisation, based on the ATS 2001 criteria, 19\% underwent a clinical failure either during hospitalisation or after discharge. Among the 410 patients who reached clinical stability within the first week of hospitalisation, based on the ATS/IDSA 2007 criteria, 17\% underwent a clinical failure either during hospitalisation or after discharge. The difference was not significant $(p=0.491)$.

The ROC curves for both ATS and ATS/IDSA criteria for clinical stability with respect to the clinical outcome are shown in figure 2. ATS 2001 criteria showed an AUC of 0.705 (95\% CI: 0.649-0.671), while ATS/IDSA 2007 criteria had an AUC of 0.714 (95\% CI: 0.657-0.771); their difference was not significant $(\mathrm{p}=0.645)$.

\section{Discussion}

This study shows a discrepancy in identifying clinical stability between the criteria recommended by the ATS in 2001 and the ATS/IDSA in 2007 in more than $60 \%$ of patients who are hospitalised because of an episode of CAP. Criteria recommended by the ATS in 2001 identify clinical stability significantly earlier than those recommended by the ATS/IDSA 2007 guidelines. Finally, ATS/IDSA 2007 criteria show a slightly, but not significantly, better performance in comparison to ATS 2001 criteria with regard to clinical outcomes at 30-day follow-up.

The definition suggested by the ATS in 2001 uses only three criteria to identify a patient with CAP who reaches clinical stability, while the definition proposed by the ATS/IDSA in 2007 includes six criteria. It is expected that as more criteria are added to the definition of clinical stability, it will become more difficult for a patient to reach all the criteria. This most likely explains our finding that the ATS 2001 criteria tend to identify clinical stability earlier in comparison to the ATS/IDSA 2007 criteria. However, as more criteria are used to define clinical stability, patients will be in a more advanced period of clinical improvement, thus, will be less likely to experience further clinical deterioration. This could explain the fact that in our population, if clinical stability during hospitalisation was defined using the ATS/IDSA 2007 criteria, fewer patients had any evidence of clinical deterioration at 30-day follow-up after discharge. 
TABLE 2 Demographics, severity of disease, clinical, laboratory, radiological findings, microbiology and treatment data of the study population

\section{Variable}

\begin{tabular}{|c|c|}
\hline Subjects & 487 \\
\hline \multicolumn{2}{|l|}{ Demographics } \\
\hline Age years & $73(61-79)$ \\
\hline Male & 477 (97.9) \\
\hline Current smoker & $204(41.9)$ \\
\hline Nursing home resident & $21(4.3)$ \\
\hline \multicolumn{2}{|l|}{ Comorbidities } \\
\hline Essential hypertension & $339(69.6)$ \\
\hline Coronary artery disease & $206(42.3)$ \\
\hline Congestive heart failure & $123(25.3)$ \\
\hline Chronic obstructive pulmonary disease & 241 (49.5) \\
\hline Cerebrovascular disease & $56(11.5)$ \\
\hline Diabetes & $177(36.3)$ \\
\hline Renal diseases & 74 (15.2) \\
\hline Immunocompromised ${ }^{\#}$ & $83(17.0)$ \\
\hline \multicolumn{2}{|l|}{ Severity of the disease on admission } \\
\hline ICU admission & 85 (17.5) \\
\hline Severe CAP & $107(22.0)$ \\
\hline CURB- 65 score 3,4 and 5 & $49(10.1)$ \\
\hline PSI risk class IV and V & $282(57.9)$ \\
\hline Altered mental status & $45(9.2)$ \\
\hline Alteration of gas exchange ${ }^{+}$ & $169(34.7)$ \\
\hline \multicolumn{2}{|l|}{ Physical findings on admission } \\
\hline Temperature ${ }^{\circ} \mathrm{F} /{ }^{\circ} \mathrm{C}$ & $99(98-101) / 37.2(36.7-38.3)$ \\
\hline Respiratory rate $\geqslant 30$ breaths $\cdot \mathrm{min}^{-1}$ & $50(10.3)$ \\
\hline Systolic blood pressure $<90 \mathrm{mmHg}$ & $22(4.5)$ \\
\hline Diastolic blood pressure $\leqslant 60 \mathrm{mmHg}$ & $32(6.6)$ \\
\hline Heart rate $\geqslant 125$ beats $\cdot \min ^{-1}$ & $51(10.5)$ \\
\hline \multicolumn{2}{|l|}{ Radiological findings } \\
\hline Multilobar infiltrates & $128(26.3)$ \\
\hline Pleural effusion & $84(17.2)$ \\
\hline \multicolumn{2}{|l|}{ Laboratory values on admission } \\
\hline $\mathrm{pH}<7.35$ & $23(4.7)$ \\
\hline Albumin $\mathrm{mg} \cdot \mathrm{L}^{-1}$ & $3.5(3.1-3.9)$ \\
\hline Creatinine $\mathrm{mg} \cdot \mathrm{dL}^{-1}$ & $1.2(0.9-1.6)$ \\
\hline White blood cells count cell $\cdot \mathrm{L}^{-1}$ & $13.1(10.3-17.2)$ \\
\hline Platelets cell $\cdot \mathrm{L}^{-1}$ & $248(197-337)$ \\
\hline Blood urea nitrogen $\mathrm{mg} \cdot \mathrm{dL}^{-1}$ & $20(14-31)$ \\
\hline Sodium $<130 \mathrm{mmol} \cdot \mathrm{L}^{-1}$ & $32(6.6)$ \\
\hline Haematocrit $<30 \%$ & $31(6.4)$ \\
\hline \multicolumn{2}{|l|}{ Microbiological profile } \\
\hline Unknown aetiology & $385(79.1)$ \\
\hline Streptococcus pneumoniae & 36 \\
\hline MRSA & 14 \\
\hline MSSA & 18 \\
\hline Pseudomonas aeruginosa & 11 \\
\hline Haemophilus influenzae & 14 \\
\hline Legionella pneumophila & 6 \\
\hline Moraxella catarralis & 6 \\
\hline Escherichia coli & 2 \\
\hline Enterobacter & 1 \\
\hline Klebsiella pneumoniae & 4 \\
\hline Pneumocystis carinii & 1 \\
\hline Serratia & 1 \\
\hline Streptococcus pyogenes & 1 \\
\hline Virus & 1 \\
\hline Other & 4 \\
\hline Mixed infection & 18 (3.7) \\
\hline Bacteraemia & 16 (3.3) \\
\hline \multicolumn{2}{|l|}{ Empirical treatment } \\
\hline Time to first antibiotic dose $\mathrm{h}$ & $7(4.5-10)$ \\
\hline Compliant to local guidelines & $405(83.2)$ \\
\hline Combination therapy & $357(73.3)$ \\
\hline
\end{tabular}

Data are presented as n, $\mathrm{n}(\%)$ or median (interquartile range) for continuous data, unless otherwise stated. ICU: intensive care unit; CAP: community-acquired pneumonia; CURB-65: confusion, urea $>7 \mathrm{mmol} \cdot \mathrm{L}^{-1}$, respiratory rate $\geqslant 30 \mathrm{breaths} \cdot \mathrm{min}^{-1}$, blood pressure $<90 \mathrm{mmHg}$ (systolic) $\leqslant 60 \mathrm{mmHg}$ (diastolic), age $\geqslant 65$ years; PSI: pneumonia severity index; MRSA: methicillin-resistant Staphylococcus aureus; MSSA: methicillin-sensitive S. aureus. ${ }^{\#}$ : active cancer, HIV or chronic steroid treatment; " ${ }^{\circ}$ : severe CAP was defined based on the American Thoracic Society/Infectious Diseases Society of America guidelines published in 2007; ${ }^{+}$: arterial oxygen tension $\left(\mathrm{PaO}_{2}\right)$ $<60 \mathrm{mmHg}$ or $\mathrm{PaO}_{2}$ /inspiratory oxygen fraction ratio $<300$ or arterial oxygen saturation measured by pulse oximetry $<90 \%$. 
TABLE 3 Joint distribution of time to clinical stability according to American Thoracic Society (ATS) 2001 and ATS/Infectious Diseases Society of America (IDSA) 2007 criteria: number of patients in the study population

Days

ATS/IDSA 2007

Total

\begin{tabular}{ccccccccccc} 
& & $\mathbf{1}$ & $\mathbf{2}$ & $\mathbf{3}$ & $\mathbf{4}$ & $\mathbf{5}$ & $\mathbf{6}$ & $\mathbf{7}$ & $\mathbf{8}$ \\
\hline \multirow{2}{*}{ ATS 2001 } & $\mathbf{1}$ & $63(12.9)$ & $28(5.7)$ & $15(3.1)$ & $7(1.4)$ & $3(0.6)$ & $2(0.4)$ & 0 & $4(0.8)$ & $122(25.1)$ \\
& $\mathbf{2}$ & $35(7.2)$ & $48(9.9)$ & $30(6.2)$ & $15(3.1)$ & $8(1.6)$ & $3(0.6)$ & $2(0.4)$ & $8(1.6)$ & $149(30.6)$ \\
& $\mathbf{3}$ & $11(2.3)$ & $17(3.5)$ & $22(4.5)$ & $15(3.1)$ & $4(0.8)$ & $2(0.4)$ & $5(1.0)$ & $5(1.0)$ & $81(16.6)$ \\
& $\mathbf{4}$ & $3(0.6)$ & $4(0.8)$ & 0 & $15(3.1)$ & $11(2.3)$ & $5(1.0)$ & $1(0.2)$ & $5(1.0)$ & $44(9.0)$ \\
& $\mathbf{5}$ & $2(0.4)$ & $3(0.6)$ & 0 & $1(0.2)$ & $3(0.6)$ & $5(1.0)$ & $1(0.2)$ & $3(0.6)$ & $18(3.7)$ \\
& $\mathbf{6}$ & 0 & 0 & $1(0.2)$ & $2(0.4)$ & 0 & 0 & $1(0.2)$ & $2(0.4)$ & $6(1.2)$ \\
& $\mathbf{7}$ & 0 & 0 & 0 & 0 & $1(0.2)$ & 0 & $3(0.6)$ & $5(1.0)$ & $9(1.8)$ \\
\multirow{2}{*}{ Total } & $\mathbf{8}$ & $3(0.6)$ & $2(0.4)$ & $1(0.2)$ & $1(0.2)$ & $3(0.6)$ & $1(0.2)$ & $2(0.4)$ & $45(9.2)$ & $58(11.9)$ \\
& & $117(24.0)$ & $102(20.9)$ & $69(14.2)$ & $56(11.5)$ & $33(6.8)$ & $18(3.7)$ & $15(3.1)$ & $77(15.8)$ & $487(100)$ \\
\hline
\end{tabular}

Data are presented as $\mathrm{n}(\%)$.

Several implications of our results can be identified from research and clinical perspectives. In the field of clinical research, when comparing time to clinical stability as the early clinical outcome in patients with CAP, both sets of clinical criteria could be applied equally. The advantage of the ATS/IDSA 2007 criteria is that they are based on objective parameters that can be easily derived from most available electronic medical records. By contrast, the ATS 2001 criteria use symptoms of pneumonia, such as cough and shortness of breath, that need to be assessed daily and then compared day-by-day. These patient-based criteria may be difficult to detect in a retrospective study. However, symptoms of pneumonia could be easily obtained in a prospective study.

In the recent document "Endpoints and Clinical Trial Issues in Community-Acquired Bacterial Pneumonia" the FDA emphasised symptom resolution as objective evidence of clinical improvement in patients with CAP [4]. Following these recommendations, it seems that the ATS 2001 criteria for clinical stability, using the improvement of subjective criteria that are directly collected from the patient, are more in-line with the FDA requirements than the ATS/IDSA 2007 criteria.

From a clinical point of view, it should be reassuring for the practicing physician that the ATS 2001 and ATS/IDSA 2007 criteria equally define clinical stability and perform similarly in hospitalised patients with CAP. During daily clinical practice, it may not be necessary to remember specific thresholds of vital parameters to define clinical stability in hospitalised patients with CAP. A patient with improving signs and symptoms of pneumonia in comparison with the previous day could be safely considered clinically stable.

Recent literature in pneumonia has identified several biomarkers, such as C-reactive protein (CRP), procalcitonin (PCT) and pro-adrenomedullin that can help manage patients with lower respiratory tract infections [13]. MENÉndez et al. [14] found that low levels of CRP and PCT, in addition to clinical criteria, seem to increase the likelihood of an absence of severe complications in hospitalised patients with CAP. Furthermore, repeat measurements of CRP after 72 or $96 \mathrm{~h}$ from initiation of antibiotic therapy seem to be helpful in identifying patients with treatment failure $[15,16]$. Future research is needed to define if the

FIGURE 1 Survival curve showing the proportion of patients who progressively reached clinical stability during the first week of hospitalisation according to both sets of criteria. ATS: American Thoracic Society; IDSA: Infectious Diseases Society of America.

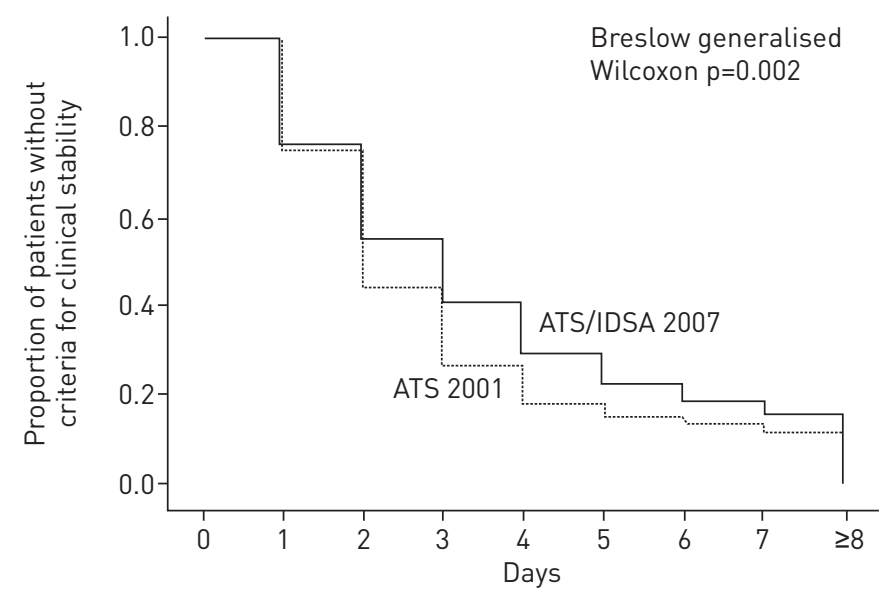


TABLE 4 Clinical outcomes of patients who reached clinical stability within 7 days from admission according to both American Thoracic Society (ATS) 2001 and ATS/Infectious Diseases Society of America (IDSA) 2007 criteria

\section{Patients}

Clinical failure during hospitalisation

Respiratory instability

Haemodynamic instability

In-hospital death

Clinical failure within $\mathbf{3 0}$ days after discharge

Re-hospitalisation within 30 days after discharge

Death within 30 days after discharge

Clinical failure either during hospitalisation or within 30 days after discharge

429
$11(2.6)$
$9(2.1)$
0
$4(0.9)$
$69(16.1)$
$62(14.5)$
$14(3.3)$
$80(18.6)$

$5(1.2)$

0

0

$64(15.6)$

$59(14.4)$

$14(3.4)$

$69(16.8)$

Data are presented as $\mathrm{n}$ or $\mathrm{n}(\%)$.

addition of serum biomarkers to the current criteria for clinical stability will be beneficial in the management of CAP patients. The advantage of current criteria for clinical stability is that they are based on signs and symptoms that could be easily obtained in every clinical setting.

Most of the original works in the field of clinical stability were focused on the development of criteria to define when a CAP patient was stable enough to be switched to oral antibiotics. When patients were followed from the time that they reached stability until the time that they were discharged from the hospital, it was found that clinical deterioration from the time of clinical stability to hospital discharge occurred in less than $2 \%$ of the cases. Based on these data, it was considered that at the point of clinical stability not only can the switch to oral therapy occur, but also it was safe to discharge the majority of patients, since clinical deterioration in the days following stability was a rare event. The original works on clinical stability did not evaluate clinical outcomes after hospital discharge. In the current study we evaluated each stability criteria for negative outcomes during hospitalisation as well as negative outcomes after discharge within 30 days. Our data evaluating 30-day outcomes indicate that both criteria have a similar rate of failure after hospital discharge. The fact that approximately $20 \%$ of patients have evidence of deterioration after hospital discharge emphasises the concept that patients reaching clinical stability will not have early deterioration, but these patients may still develop clinical deterioration after hospital discharge. Data from GuerTLER et al. [17] clearly showed that significant medical conditions, such as chronic obstructive pulmonary disease, cardiac disease and malignancy, are some of the reasons behind adverse outcomes after discharge in CAP patients. The majority of clinical failures after discharge in our population were due to re-hospitalisations. Our data are inline with those reported by recent literature [18-21]. In particular, JASTI et al. [18] found that 12\% of patients discharged after hospitalisation for CAP were re-hospitalised within 30 days and most of these were comorbidity-related and not pneumonia-related. Our findings suggest that current criteria for clinical stability, as windows of the early response to infection and inflammation, cannot predict the subsequent impact of the pneumonia on the deterioration of comorbidities after discharge that could be responsible for both deaths and readmissions. At the moment, the time in which a CAP patient reaches clinical stability

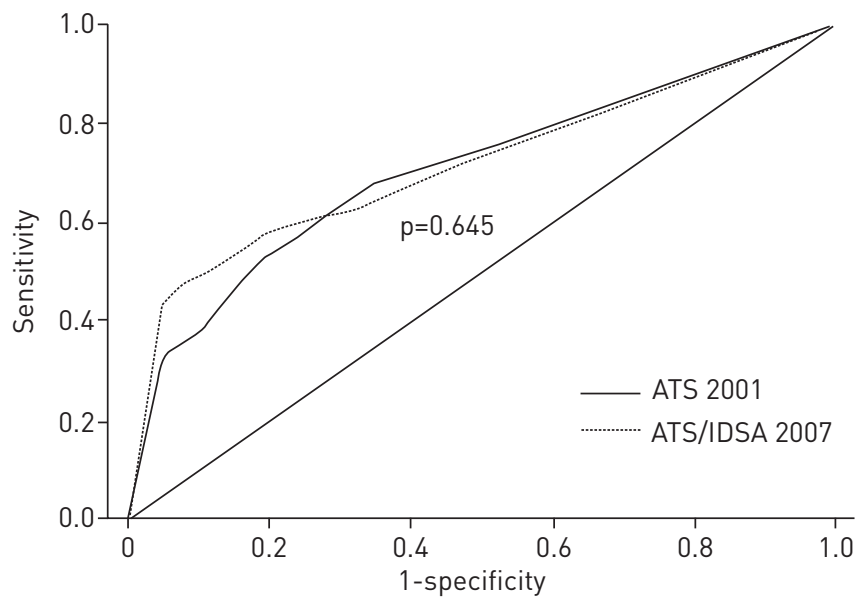

FIGURE 2 Receiver operating characteristic curves for American Thoracic Society (ATS) 2001 and ATS/Infectious Diseases Society of America (IDSA) 2007 criteria with respect to the clinical failure either during hospitalisation or after discharge. 
during hospitalisation, in addition to the presence of signs of instability at discharge, seems to be the only tool that can help physicians in predicting adverse outcomes after discharge [20,22].

One important limitation of our study is its retrospective design. Furthermore, the population belonged to a single hospital and was composed primarily of elderly people, particularly males, with a markedly high number of comorbidities. This may affect the generalisability of our findings. Our study is strengthened by the evaluation of the two most used criteria for clinical stability among the same population of consecutive patients hospitalised for an episode of CAP.

In conclusion, we identified only minimal differences in the performances of ATS 2001 and ATS/IDSA 2007 criteria in defining clinical stability in hospitalised patients with CAP. Both criteria can identify early clinical stability and can be used in clinical practice as well as in clinical trials of patients with CAP.

\section{Acknowledgements}

The authors acknowledge the assistance of Mehdi Mirsaeidi and Asad Amir (Division of Infectious Diseases, University of Louisville, Louisville, KY, USA), and Elizabeth Smigielski (Kornhauser Health Sciences Library, University of Louisville, Louisville, KY, USA).

\section{References}

1 Niederman MS, Mandell LA, Anzueto A, et al. Guidelines for the management of adults with community-acquired pneumonia. Diagnosis, assessment of severity, antimicrobial therapy, and prevention. Am J Respir Crit Care Med 2001; 163: 1730-1754

2 Ramirez JA, Srinath L, Ahkee S, et al. Early switch from intravenous to oral cephalosporins in the treatment of hospitalized patients with community-acquired pneumonia. Arch Intern Med 1995; 155: 1273-1276.

3 Ramirez JA, Vargas S, Ritter GW, et al. Early switch from intravenous to oral antibiotics and early hospital discharge: a prospective observational study of 200 consecutive patients with community-acquired pneumonia. Arch Intern Med 1999; 159: 2449-2454.

4 Division of Anti-infective Products/Office of Antimicrobial Products. Anti-Infective Drugs Advisory Committee. Endpoints and Clinical Trial Issues in Community-Acquired Bacterial Pneumonia. www.fda.gov/downloads/ advisorycommittees/committeesmeetingmaterials/drugs/anti-infectivedrugsadvisorycommittee/ucm275823.pdf Date last accessed: May 15, 2012. Date last updated: November 3, 2011.

5 Musher DM. Clinical and microbiological end points in the treatment of pneumonia. Clin Infect Dis 2008; 47: Suppl. 3, S207-S209.

6 Mandell LA, Wunderink RG, Anzueto A, et al. Infectious Diseases Society of America/American Thoracic Society consensus guidelines on the management of community-acquired pneumonia in adults. Clin Infect Dis 2007; 44: Suppl. 2, S27-S72.

7 Halm EA, Fine MJ, Marrie TJ, et al. Time to clinical stability in patients hospitalized with community-acquired pneumonia: implications for practice guidelines. JAMA 1998; 279: 1452-1457.

8 Ramirez JA. Fostering international multicenter collaborative research: the CAPO Project. Int J Tuberc Lung Dis 2007; 11: 1062-1065.

9 Aliberti S, Amir A, Peyrani P, et al. Incidence, etiology, timing, and risk factors for clinical failure in hospitalized patients with community-acquired pneumonia. Chest 2008; 134: 955-962.

10 Fine MJ, Auble TE, Yealy DM, et al. A prediction rule to identify low-risk patients with community-acquired pneumonia. N Engl J Med 1997; 336: 243-250.

11 Lim WS, Lewis S, Macfarlane JT. Severity prediction rules in community-acquired pneumonia: a validation study. Thorax 2000; 55: 219-223.

12 DeLong ER, DeLong DM, Clarke-Pearson DL. Comparing the areas under two or more correlated receiver operating characteristic curves: a nonparametric approach. Biometrics 1988; 44: 837-845.

13 Schuetz P, Amin DN, Greenwald JL. Role of procalcitonin in managing adult patients with respiratory tract infections. Chest 2012; 141: 1063-1073.

14 Menéndez R, Martinez R, Reyes S, et al. Stability in community-acquired pneumonia: one step forward with markers? Thorax 2009; 64: 987-992.

15 Gómez J, Baños V, Ruiz Gómez J, et al. Prospective study of epidemiology and prognostic factors in communityacquired pneumonia. Eur J Clin Microbiol Infect Dis 1996; 15: 556-560.

16 Menéndez R, Cavalcanti M, Reyes S, et al. Markers of treatment failure in hospitalised community acquired pneumonia. Thorax 2008; 63: 447-452.

17 Guertler C, Wirz B, Christ-Crain M, et al. Inflammatory responses predict long-term mortality risk in communityacquired pneumonia. Eur Respir J 2011; 37: 1439-1446.

18 Jasti H, Mortensen EM, Obrosky DS, et al. Causes and risk factors for rehospitalization of patients hospitalized with community-acquired pneumonia. Clin Infect Dis 2008; 46: 550-556.

19 Halm EA, Fine MJ, Kapoor WN, et al. Instability on hospital discharge and the risk of adverse outcomes in patients with pneumonia. Arch Intern Med 2002; 162: 1278-1284.

20 Capelastegui A, España PP, Bilbao A, et al. Pneumonia: criteria for patient instability on hospital discharge. Chest 2008; 134: 595-600.

21 Capelastegui A, España PP, Quintana JM, et al. Declining length of hospital stay for pneumonia and postdischarge outcomes. Am J Med 2008; 121: 845-852.

22 Aliberti S, Peyrani P, Filardo G, et al. Association between time to clinical stability and outcomes after discharge in hospitalized patients with community-acquired pneumonia. Chest 2011; 140: 482-488. 